\title{
Cooperative Contractility: the Role of Stress Fibres in the Regulation of Cell-Cell JUNCTIONS
}

William Ronan ${ }^{a, b^{\dagger}}$, Robert M. McMeeking ${ }^{c, d}$, Christopher S. Chen ${ }^{e, f}$, J. Patrick McGarry ${ }^{a}$, Vikram S. Deshpande ${ }^{b}$

a

Department of Mechanical and Biomedical Engineering, , National University of Ireland Galway, University Road, Galway, Ireland.

b Department of Engineering, University of Cambridge, Trumpington Street, Cambridge CB2 1PZ, UK.

c Departments of Mechanical Engineering and Materials., University of California, Santa Barbara, CA 93106-5070, U.S.A.

d School of Engineering, University of Aberdeen King's College, Aberdeen AB24 3UE, Scotland

e Department of Biomedical Engineering, Boston University, Boston, MA, USA

f Wyss Institute for Biologically Inspired Engineering, Harvard University, Boston, MA, USA

† corresponding author email:wr247@eng.cam.ac.uk

Keywords: mechanotransduction; stress fibre; junction force; myosin contractility; cell-cell junctions.

Submitted as an Original Article to the Journal of Biomechanics

Word count (Introduction to Conclusions) : 3461 


\begin{abstract}
We present simulations of cell-cell adhesion as reported in a recent study [Liu et al., 2010, PNAS, 107(22), 9944-9] for two cells seeded on an array of micro-posts. The micro-post array allows for the measurement of forces exerted by the cell and these show that the cell-cell tugging stress is a constant and independent of the cell-cell junction area. In the current study, we demonstrate that a material model which includes the underlying cellular processes of stress fibre contractility and adhesion formation can capture these results. The simulations explain the experimentally observed phenomena whereby the cell-cell junction forces increase with junction size but the tractions exerted by the cell on the micro-post array are independent of the junction size. Further simulations on different types of micro-post arrays and cell phenotypes are presented as a guide to future experiments.
\end{abstract}




\section{Introduction}

While many investigations have been performed on the mechano-sensing role of focal adhesions (FA), the properties of cell-cell junctions are less well understood. FA mechanics and biochemistry have been extensively studied for single cells adhering to a variety of substrates: continuous rigid and compliant substrates (Discher et al., 2005; Elineni and Gallant, 2011), micro-patterned islands and micro-post arrays (Dalby et al., 2007; Tan et al., 2003; Théry et al., 2006), as well as fibrous constructs (Fraley et al., 2010). The study of cell-cell adhesion is complicated by the need to study a cell population, which inhibits accurate measurement of the force across a particular cell-cell interface. Micro-beads, pipettes, or AFM cantilevers have previously been coated with cadherin in order to mimic the surface of another cell and thus artificially form a cell-cell junction. However, these systems do not capture the dynamic interactions between the cells forming a junction, which may cooperatively or independently generate tractions.

A recent study by Liu et al. (2010) has developed a novel experimental system to study cell-cell adhesion using traction microscopy techniques. Previous studies have used arrays of micro-posts, or micro-pillars, to measure the contractile response of cells (Kural and Billiar, 2014; Tan et al., 2003) and quantify the relationship between traction and FA area (Fu et al., 2010; Tan et al., 2003). Liu et al. (2010) use an array of micro-posts that are selectively coated with fibronectin to restrict the shape of two neighbouring cells such that they adhere to form a "bowtie" shape, as shown in Figure 1. The net force generated by each cell on the junction is then calculated from the individual post deflections as described in Liu et al. (2010).

Computational models for eukaryotic cells have focussed primarily on their passive properties to include the properties of the cytoplasm and the passive meshwork of fibres (Nelson et al., 2005; Satcher Jr and Dewey Jr, 1996; Storm et al., 2005; Unterberger et al., 2013). Models for the active response of cells have historically been restricted to muscle cells; see for example a recent study by Stålhand et al. (2011). The contractile properties of non-muscle cells due to the activity of stress fibres (SFs) have received increasing attention with the advent of experimental techniques such as micro-post arrays that allow the measurement of cellular forces (Elson and Genin, 2013). Computational models for SFs include networks models where filaments with prescribed shrinkage strains are specified (Mohrdieck et al., 2005). Such approaches neglect the biochemistry of the active apparatus of the cell that generates, supports and responds to mechanical forces. The so-called tensegrity model (Ingber, 1993) has previously been used to simulate cells on elastic substrates (De Santis et al., 2011); however, the tensegrity model requires a predefined cytoskeleton. Furthermore, it has been shown experimentally that the disruption of microtubules results in an increase in the traction force generated 
by cells (Kolodney and Elson, 1995), contradicting the central assumption of the tensegrity model. Deshpande et al. (2006) developed a framework that captures the signal and tension dependent remodelling of SFs. This approach has been extended in numerous studies; see for example Kaunas and Hsu (2009) and Obbink-Huizer et al. (1996) whose models better account for the remodelling of cells subjected to cyclic stretch. Modelling of focal adhesions (FAs) (Unterberger et al., 2013) and cell-cell adhesions through cadherins (Stålhand et al., 2011) have focussed on the properties of the adhesive proteins but neglected their coupling to cell contractility. Deshpande et al. (2008) attempted to overcome this shortcoming by proposing a thermodynamically motivated model that accounts for the co-operativity between focal adhesion formation and cell contractility.

The experiments of Liu et al. (2010) investigated the relation between cell traction forces and cell-cell tugging forces through the cell-cell junctions. They employed a bow-tie arrangement of cell pairs to facilitate relatively large changes in junction area, while keeping cell area and focal adhesion area constant (i.e. the number of attached micro-posts). This experimental design thus isolates cell-cell adhesion behaviour from the other mechano-sensitive processes. In the current study we extend the frameworks of Deshpande et al. (2006) and (2008) to investigate the behaviour of cells that have formed cell-cell junctions. We restrict our attention to the bowtie cell geometries so as to be able to independently vary junction and focal adhesion area similar to the experiments of Liu et al. (2010). Our simulations uncover the mechanisms underlying the experimentally observed phenomena and elucidate the relationships between contractility, junction tugging force, and micro-post tractions. We present this study in two sections: first, we simulate the behaviour of bowtie shaped cell pairs and validate our model based on the experimental results of Liu et al. (2010); and second, we investigate the effect of parameters such as micro-post stiffness, cell phenotype, and post array geometry on the cell-cell adhesion response.

\section{Methods}

\subsection{Modelling approach}

Our computational model replicates the key components of the experiments of Liu et al. (2010). The four main aspects of the model are described briefly here and a detailed description is given in Appendix A.

(1) The micro-post array consists of PDMS pillars, which behave like upright cantilevered beams (Figure 1C). For small beam deflections, the micro-post tip force $\left|\boldsymbol{F}_{\boldsymbol{p}}\right|$ is proportional to the tip displacement $\left|\boldsymbol{\delta}_{\boldsymbol{p}}\right|$ (vectors are represented 
in boldface). Thus, it suffices to only model the top circular surface of the post that the cell is adhered to, and represent the bending stiffness of the post via a linear spring.

(2) The response of the cell is governed by the contractile behaviour of actin stress fibres (SFs), which are surrounded by the cytoplasm and other cellular components. The active contractility of the SFs due to cross bridge cycling of myosin is captured in the material formulation (Deshpande et al., 2006) using a Hill-like tension-velocity law. The bio-chemo-mechanical remodelling of the SFs is captured via a kinetic model whereby SFs form in response to a signal and dissociate when fibre tension falls below the isometric level. Our model does not assume any a priori SF distribution: the cell is initially fibre-free. SFs form in response to an activation signal and only persist where there is sufficient support for fibre tension. Therefore, the SF distribution is predicted by the model and depends on the stiffness of the micro-posts, the arrangement of the cells relative to the micro-posts, and cell-cell adhesion. Subsequent changes in externally applied loads will thus lead to further remodelling of the SF network.

(3) The cells adhere to the micro-posts, and in general to other substrates or ECM, via mechano-sensitive focal adhesions (FAs). In the current study, the formation of FAs, via the binding of integrins to suitable ligands on the posts, is captured with a thermodynamically motivated model (Deshpande et al., 2008). This model considers the thermodynamic balance between (i) the stretching of bonds and (ii) integrins switching from low energy, unbound states to high energy, bound states. This leads to tension dependent FA formation, whereby increasing traction increases the concentration of bound integrins and, consequently, the stiffness of the adhesion. We note in passing that FA distributions have been previously studied by the authors (Pathak et al., 2008; Ronan et al., 2013) and, hence, we do not present details of FA distributions in the current study.

(4) Finally, cell-cell adhesion occurs via the binding of cadherin from each cell. The thermodynamic FA model is adapted to consider bond formation between cadherin instead of between integrins and ligands. Therefore, cell-cell adhesion formation is also tension dependent.

The simulations presented here focus on the steady-state response of a contractile cell pair and the predictions are insensitive to relative kinetics of the different processes such as signal development, stress fibre growth, and the turnover of the integrin and cadherin adhesion proteins. We note that there exists a large body of experimental data on the kinetics of these cellular processes but capturing the transient behaviours due to these kinetics is beyond the scope of the current study.

\subsection{Finite element simulations}


The material and adhesion formulations described in Appendix A are included in the finite element program Abaqus (v6,12, Dassault Systemes, RI, USA) as a user-defined material (UMAT) and a user-defined interaction (UINTER) respectively. Exploiting symmetry, the finite element model consists of half of one of the cells with the underlying posts, as shown in Figure 2A. The two dimensional simulations (cell of thickness $5 \mu \mathrm{m}$ perpendicular to the plane of Figure 2) consist of a single analysis step, where SFs form in response to an exponentially decaying activation signal. SF formation and reorganisation is simulated for $700 \mathrm{~s}$, by which time a steady state distribution has formed.

The material and contact parameters are based on previous calibrations of this model (McGarry et al., 2009; Pathak et al., 2008; Reynolds et al., 2014). The parameters for the adhesion model are the same for all cell types: $\mu_{B}-\mu_{U}=2.14$ $10^{-5} \mathrm{fJ} ; \xi_{0}=500 \mu \mathrm{m}^{-2} ; T=310 \mathrm{~K} ; \kappa_{s}=0.015 \mathrm{nN} / \mu \mathrm{m} ; \Delta_{n}=0.13 \mu \mathrm{m}$. Similarly, the cell model parameters which are common to all cell types are: $\dot{\varepsilon_{0}}=0.003 \mathrm{~s}^{-1} ; \theta=70 \mathrm{~s} ; \overline{k_{f}}=10 ; \overline{k_{b}}=1$. Moreover, for all cell types, the Young's modulus $E_{c e l l}$ and Poisson's ratio $v_{c e l l}$ of the cell, which represent the passive components of the cytoplasm and nucleus, were fixed at $0.4 \mathrm{kPa}$ and 0.3 , respectively. Four different cell phenotypes are considered: mesenchymal stem cells (MSCs), fibroblasts (FBs), smooth muscle cells (SMCs), and endothelial cells (ETCs). For each of these, $\sigma_{\max }$ and $\overline{k_{v}}$ are altered to represent the different levels of contractility as listed in Table 1.

\subsection{Interpretation of results}

The individual post forces can be determined from the post deflections, as described in Appendix A. The total $\left(F_{t o t}\right)$ and average $\left(F_{\text {avg }}\right)$ micro-post traction forces then are calculated as

$$
F_{t o t}=\sum_{m=1}^{N}\left|\boldsymbol{F}_{p}\right|_{m} \text { and } \quad F_{a v g}=\frac{F_{t o t}}{N}
$$

respectively, where $\left|\boldsymbol{F}_{p}\right|_{m}$ is the magnitude of the force vector exerted by the cell on post $m$ and $N$ is the total number of micro-posts adhered to the cell. The force at the cell-cell junction $\boldsymbol{F}_{J}$ is determined from the resultant of all the individual post forces, as in the experiments of Liu et al. (2010) via the relation

$$
\boldsymbol{F}_{J}=-\sum_{m=1}^{N}\left(\boldsymbol{F}_{p}\right)_{m}
$$

and the cell-cell junction stress then defined as $\left|\boldsymbol{F}_{J}\right| / A_{J}$, where $A_{J}$ is the junction area (junction width multiplied by cell height). The SF distributions are visualised by plotting the difference between the maximum level of SF activation and 
the mean level at a point, which we call the SF variance. The average level of SF formation at a point is calculated by integrating the level of SF formation over every possible direction $\phi$ (Figure 2)

$$
\bar{\eta} \equiv \frac{1}{\pi} \int_{-\pi / 2}^{\pi / 2} \eta d \phi
$$

The variance $\Pi$ is then calculated at each point by subtracting this average from the maximum level of SF formation in any direction:

$$
\Pi=\eta_{\max }-\bar{\eta}
$$

\section{Predictions of the response of the cell pairs in the experiments of Liu et al. (2010)}

Simulations of cell-cell adhesion, based on the experiments on endothelial cells of Liu et al. (2010), are presented in Figure 3. Two triangular shaped cells are arranged to form a bow-tie with a cell-cell junction, i.e. at the centre of the bow-tie. Four different configurations are considered with different junction cross-sectional areas $\left(A_{J}=12.5,25,50\right.$, and $75 \mu \mathrm{m}^{2}$ ) with the post spacing and post-diameter equal to $\sim 10 \mu \mathrm{m}$ and $3 \mu \mathrm{m}$ respectively as in the experiments of Liu et al. (2010). The computed tugging force across the junction increases linearly with increasing junction size (Figure 3A). In contrast, the average traction force $F_{a v g}$, i.e. the average of the magnitudes of the individual post forces, is independent of junction size (Figure 3B). These predictions are in excellent agreement with the experimental observations of Liu et al. (2010), which are superimposed over the computed results in Figure 3A,B. The linear relationship between junction size and junction tugging force $\left|\boldsymbol{F}_{J}\right|$ results in an approximately constant junction stress of approximately $1 \mathrm{kPa}$ for both the simulations and experimental data.

Figure $3 C$ shows stress fibre (SF) formation in cell pairs for different junction areas $A_{J}$. SF formation in the cell away from the junction is unaffected by the size of the junction and, hence, the average traction force is unaffected by the junction size. However, an increase in junction size is accompanied by an increase in SF formation in the region immediately adjacent to the junction resulting in the observed increase in the tugging force $\left|\boldsymbol{F}_{J}\right|$.

\section{Cell pair behaviour on different micro-post arrays}


Previous studies of individual cells on micro-post arrays have investigated the effect of micro-post stiffness (Fu et al., 2010; McGarry et al., 2009; Tee et al., 2011) micro-post spacing and size (Yang et al., 2007) and cell phenotype (Fu et al., 2010; McGarry et al., 2009). However, the study of Liu et al. (2010) on cell pairs used only one micro-post array (stiffness $=32 \mathrm{nN} / \mu \mathrm{m}$, post spacing $=\sim 10 \mu \mathrm{m}$, and post diameter $=3 \mu \mathrm{m}$ ) for endothelial cells. In this section, we present numerical predictions to investigate the sensitivity of post stiffness and arrangement on the observations of Liu et al. (2010) for the endothelial cells.

\subsection{Micro-post stiffness affects traction but not tugging}

Increasing micro-post stiffness $k_{p}$ for cell pairs causes an increase in SF formation throughout the cells, as shown in Figure 4. However, it should be noted that for all but the most compliant posts, there are high levels of SFs in the area between the post closest to the junction and junction itself. These SF patterns result in a monotonic increase in average traction $F_{\text {avg }}$ with increasing stiffness, but with an almost unchanged junction tugging force $\left|\boldsymbol{F}_{J}\right|$ for micro-posts stiffer than $3.2 \mathrm{nN} / \mu \mathrm{m}$ and a rapid decrease for more compliant micro-posts.

The linear relationship between junction tugging force and junction size observed by Liu et al. (2010) (and captured by the simulations), breaks-down for very compliant micro-posts $(<1 \mathrm{nN} / \mu \mathrm{m})$, as shown in Figure $4 \mathrm{C}$. Instead of maintaining a constant junction stress of $\sim 1 \mathrm{kPa}$, as observed for the $32 \mathrm{nN} / \mu \mathrm{m}$ micro-post array, compliant micro-posts lead to a constant junction force for all simulated junction sizes and thus a decrease in junction stress with increasing $A_{J}$. Increasing micro-post stiffness by a factor of 10 to $320 \mathrm{nN} / \mu \mathrm{m}$ from $32 \mathrm{nN} / \mu \mathrm{m}$ does not significantly change the junction stress from that measured by Liu et al. (2010). The average traction is predicted to decrease with micro-post stiffness and to be independent of junction size (Figure 4D).

The results in Figure 4C,D clearly show that $\left|\boldsymbol{F}_{J}\right|$ and $F_{a v g}$ are decoupled (i.e. $\left|\boldsymbol{F}_{J}\right|$ increases linearly with $A_{J}$, but $F_{a v g}$ is unaffected by $A_{J}$ ). To better understand this, consider the single cell (Figure 5A) and cell pair (Figure 5B) on a bed of $3.2 \mathrm{nN} / \mu \mathrm{m}$ micro-posts. The average traction force $F_{\text {avg }}$ in both cases are nearly identical (Figure 4D). However, in the cell pairs there is also a junction force of $50 \mathrm{nN}$ (Figure $4 \mathrm{C}$ ), which is $\sim 10$ times the average traction force, acting on each cell. This counterintuitive response is explained by the orientation of the post forces (i.e. the direction in which the post is deflected). For the single cell in Figure 5A, the forces are all seen to point towards the centre of the cell; however, for the cell pair, the forces all act in the direction of the junction. This arrangement allows the average traction to remain constant while the junction tugging force, which is the net resultant of all the post forces, changes. Similarly, while comparing Figures $5 B$ and 5D, we observe that for cells pairs on a bed of $320 \mathrm{nN} / \mu \mathrm{m}$ micro-posts, the post forces act 
towards the centre of the cell rather than towards the junction. Thus, with increasing post stiffness, $F_{a v g}$ increases but $\left|F_{J}\right|$, which depends on the vector sum of the forces, remains reasonably unchanged (as shown previously in Figure 4D). Given the importance of orientation and distribution of the individual post forces, we speculate that the relationship between average traction and junction tugging forces observed in these bow-tie shaped arrangements may not persist for other cell geometries.

\subsection{Micro-post spacing decreases traction - tugging unchanged}

The effect of micro-post array designs was investigated by changing the number of micro-posts supporting each of the cells but keeping the post arrangement the same as in Liu et al. (2010); i.e. the close-packed arrangement as seen in Figure 1. Moreover, as the number of posts $N$ was increased, the diameter of the posts was reduced such that the total available adhesion area (i.e. the sum of all post-top areas) was kept constant. Consequently, it was necessary to decrease the height of the micro-posts so that the stiffness of each post remained constant $\left(k_{p}=32 \mathrm{nN} / \mu \mathrm{m}\right)$. In addition, the junction area was held fixed at $A_{J}=50 \mu \mathrm{m}^{2}$. Increasing the number of micro-posts did not cause a significant change in the total traction force $F_{\text {tot }}$ (Figure 6A) or the tugging force $\left|\boldsymbol{F}_{J}\right|$ (Figure 6C). However, as $F_{\text {tot }}$ was constant, $F_{\text {avg }}$ decreases with increasing number of micro-posts $\mathrm{N}$ (Figure 6B). SFs are not predicted to change significantly with increasing number of micro-posts (Figure 6D), with SFs mostly at the periphery of the cell and few SFs in the interior.

\section{Phenotype and contractility of cell pairs}

Simulations are reported here to investigate the effect of cell phenotype on the response of cell pairs on the micro-post array used in the experiments of Liu et al. (2010), with a constant junction size $\left(A_{J}=50 \mu \mathrm{m}^{2}\right)$. The different cell phenotypes analysed and their properties are listed in Table 1.

Increased levels of SF contractility associated with different cell phenotypes cause an increase in both average traction force and junction tugging force (Figure 7). The trends observed previously for ETCs are preserved for SMCs, MCS, and FBs. Different patterns of SFs are computed for each cell type (Figure 7A) with SFs for the most contractile cells becoming relatively dominant around the junction and lower near the interior of the cell. Despite the decrease in aligned SFs seen for SMCs, average tractions are highest for this cell type as the maximum possible SF tension is approximately 10 times higher for SMCs compared to ETCs.

\section{Concluding remarks}


In the current study, we demonstrate the ability of our computational framework to accurately predict the experimental observations of Liu et al. (2010). Junction tugging forces increase with junction size such that a constant junction stress is maintained (approx. $1 \mathrm{nN} / \mu \mathrm{m}^{2}$ ) and average micro-post tractions are unaffected by the presence of the junction. In the latter part of the study, we elucidate the role of a number of experimental parameters not considered by Liu et al. (2010), such as micro-post stiffness, cell phenotype, and micro-post array geometry. These investigations uncover how micro-post tractions are distributed and thus explain the seemingly contradictory observation of increased cell-cell junction tugging forces with unchanged average tractions.

\section{Acknowledgements}

WR and PMcG acknowledge Science Foundation Ireland grant 10/RFP/ENM2960 and Short Term Travel Fellowship (STTF 11). 


\section{References}

Dalby, M.J., Gadegaard, N., Tare, R., Andar, A., Riehle, M.O., Herzyk, P., Wilkinson, C.D.W., Oreffo, R.O.C., 2007. The control of human mesenchymal cell differentiation using nanoscale symmetry and disorder. Nat Mater 6, 997-1003.

De Santis, G., Lennon, A., Boschetti, F., Verhegghe, B., Verdonck, P., Prendergast, P., 2011. How can cells sense the elasticity of a substrate?: an analysis using a cell tensegrity model. European cells \& materials 22, 202-213.

Deshpande, V.S., McMeeking, R.M., Evans, A.G., 2006. A bio-chemo-mechanical model for cell contractility. Proceedings of the National Academy of Sciences 103, 14015-14020.

Deshpande, V.S., Mrksich, M., McMeeking, R.M., Evans, A.G., 2008. A bio-mechanical model for coupling cell contractility with focal adhesion formation. Journal of the Mechanics and Physics of Solids 56, 1484-1510.

Discher, D.E., Janmey, P., Wang, Y.-I., 2005. Tissue Cells Feel and Respond to the Stiffness of Their Substrate. Science 310, 1139-1143.

Elineni, Kranthi K., Gallant, Nathan D., 2011. Regulation of Cell Adhesion Strength by Peripheral Focal Adhesion Distribution. Biophys J 101, 2903-2911.

Elson, E.L., Genin, G.M., 2013. The role of mechanics in actin stress fiber kinetics. Exp Cell Res 319, 2490-2500.

Fraley, S.I., Feng, Y., Krishnamurthy, R., Kim, D.-H., Celedon, A., Longmore, G.D., Wirtz, D., 2010. A distinctive role for focal adhesion proteins in three-dimensional cell motility. Nature cell biology 12, 598-604.

Franke, R.P., Grafe, M., Schnittler, H., Seiffge, D., Mittermayer, C., Drenckhahn, D., 1984. Induction of human vascular endothelial stress fibres by fluid shear stress. Nature 307, 648-649.

Fu, J., Wang, Y.-K., Yang, M.T., Desai, R.A., Yu, X., Liu, Z., Chen, C.S., 2010. Mechanical regulation of cell function with geometrically modulated elastomeric substrates. Nat Meth 7, 733-736.

Ingber, D.E., 1993. Cellular tensegrity: defining new rules of biological design that govern the cytoskeleton. Journal of Cell Science 104, 613-613.

Kaunas, R., Hsu, H.J., 2009. A kinematic model of stretch-induced stress fiber turnover and reorientation. J Theor Biol 257, 320-330.

Kolega, J., 1986. Effects of mechanical tension on protrusive activity and microfilament and intermediate filament organization in an epidermal epithelium moving in culture. J Cell Biol 102, 1400-1411.

Kolodney, M.S., Elson, E.L., 1995. Contraction due to microtubule disruption is associated with increased phosphorylation of myosin regulatory light chain. Proceedings of the National Academy of Sciences 92, 10252-10256.

Kural, M.H., Billiar, K.L., 2014. Mechanoregulation of valvular interstitial cell phenotype in the third dimension. Biomaterials 35, 1128-1137.

Liu, Z., Tan, J.L., Cohen, D.M., Yang, M.T., Sniadecki, N.J., Ruiz, S.A., Nelson, C.M., Chen, C.S., 2010. Mechanical tugging force regulates the size of cell-cell junctions. Proceedings of the National Academy of Sciences 107, 9944.

McGarry, J.P., Fu, J., Yang, M.T., Chen, C.S., McMeeking, R.M., Evans, A.G., Deshpande, V.S., 2009. Simulation of the contractile response of cells on an array of micro-posts. Philosophical Transactions of the Royal Society a-Mathematical Physical and Engineering Sciences 367, 3477-3497.

Mohrdieck, C., Wanner, A., Roos, W., Roth, A., Sackmann, E., Spatz, J.P., Arzt, E., 2005. A theoretical description of elastic pillar substrates in biophysical experiments. Chemphyschem 6, 1492-1498.

Nelson, C.M., Jean, R.P., Tan, J.L., Liu, W.F., Sniadecki, N.J., Spector, A.A., Chen, C.S., 2005. Emergent patterns of growth controlled by multicellular form and mechanics. P Natl Acad Sci USA 102, 11594-11599.

Pathak, A., Deshpande, V.S., McMeeking, R.M., Evans, A.G., 2008. The simulation of stress fibre and focal adhesion development in cells on patterned substrates. J R Soc Interface 5, 507-524.

Reynolds, N.H., Ronan, W., Dowling, E.P., Owens, P., McMeeking, R.M., McGarry, J.P., 2014. On the role of the actin cytoskeleton and nucleus in the biomechanical response of spread cells. Biomaterials 35, 4015-4025.

Ronan, W., Deshpande, V.S., McMeeking, R.M., McGarry, J.P., 2012. Numerical investigation of the active role of the actin cytoskeleton in the compression resistance of cells. Journal of the Mechanical Behavior of Biomedical Materials 14, 143-157.

Ronan, W., Pathak, A., Deshpande, V.S., McMeeking, R.M., McGarry, J.P., 2013. Simulation of the mechanical response of cells on micro-post substrates. Journal of Biomechanical Engineering.

Satcher Jr, R.L., Dewey Jr, C.F., 1996. Theoretical estimates of mechanical properties of the endothelial cell cytoskeleton. Biophys J 71, 109-118.

Stålhand, J., Klarbring, A., Holzapfel, G.A., 2011. A mechanochemical 3D continuum model for smooth muscle contraction under finite strains. J Theor Biol 268, 120-130.

Storm, C., Pastore, J.J., MacKintosh, F.C., Lubensky, T.C., Janmey, P.A., 2005. Nonlinear elasticity in biological gels. Nature 435, 191-194. 
Tan, J.L., Tien, J., Pirone, D.M., Gray, D.S., Bhadriraju, K., Chen, C.S., 2003. Cells lying on a bed of microneedles: an approach to isolate mechanical force. Proc Natl Acad Sci U S A 100, 1484-1489.

Tee, S.-Y., Fu, J., Chen, Christopher S., Janmey, Paul A., 2011. Cell Shape and Substrate Rigidity Both Regulate Cell Stiffness. Biophys J 100, L25-L27.

Théry, M., Pépin, A., Dressaire, E., Chen, Y., Bornens, M., 2006. Cell distribution of stress fibres in response to the geometry of the adhesive environment. Cell Motility and the Cytoskeleton 63, 341-355.

Unterberger, M.J., Schmoller, K.M., Bausch, A.R., Holzapfel, G.A., 2013. A new approach to model cross-linked actin networks: multi-scale continuum formulation and computational analysis. Journal of the Mechanical Behavior of Biomedical Materials 22, 95-114.

Yang, M.T., Sniadecki, N.J., Chen, C.S., 2007. Geometric Considerations of Micro- to Nanoscale Elastomeric Post Arrays to Study Cellular Traction Forces. Advanced Materials 19, 3119-3123. 


\section{Appendix}

This appendix provides a brief description of the cell contractility and adhesion models. Readers are referred to the original publications by Deshpande et al. (Deshpande et al., 2006; Deshpande et al., 2008) for a more complete description.

\section{Cell contractility model}

In the current study, a cell material model that considers the contractility and remodelling of the actin cytoskeleton is used. This model was originally presented by Deshpande et al. (2006) and subsequently used to simulate both planar and complex 3D cell geometries (Pathak et al., 2008; Ronan et al., 2012). The actin-myosin cytoskeleton consists of contractile stress fibres (SFs) and three key SF phenomena are captured in the formulation: (i) formation of SFs in response to cellular signalling; (ii) dissociation of SFs following a reduction in fibre tension; (iii) and a Hill-Huxley like contractility law that relates SF tension to strain rate. First, we consider the behaviour of a single bundle of SFs.

The complex cell signalling pathways are not considered and the activation signal $C$ is phenomenologically represented as a spatially homogenous signal that decays exponentially with time

$$
C=e^{\left(\frac{-t_{1}}{\theta}\right)}
$$

where $\theta$ is a constant and $t_{1}$ is the time since the most recent signalling event.

The dimensionless activation level of a SF bundle is described by the parameter $(0 \leq \eta \leq 1)$. The Hill-Huxley like contractility relationship, whereby SF tension decreases with increasing contractile velocity (Franke et al., 1984; Kolega, 1986), is captured using a tri-linear equation. For positive strain rates, the SFs remain at the isometric tension level. For negative strain rates there are two regimes; large negative strain rates lead to complete loss of tension, and for smaller negative strain rates the tension decreases linearly with increasing contractile strain rate. This is described via the relations

$$
\frac{\sigma_{f}}{\sigma_{0}}=\left\{\begin{array}{cc}
0 & \frac{\dot{\varepsilon}_{f}}{\dot{\varepsilon_{0}}} \leq-\frac{\eta}{\overline{k_{v}}} \\
1+\frac{\overline{k_{v}}}{\eta} \frac{\dot{\varepsilon}_{f}}{\dot{\varepsilon_{0}}} & -\frac{\eta}{\overline{k_{v}}} \leq \frac{\dot{\varepsilon}_{f}}{\dot{\varepsilon}_{0}} \leq 0 \\
1 & \frac{\dot{\varepsilon}_{f}}{\dot{\varepsilon_{0}}}>0
\end{array}\right.
$$


where $\sigma_{f}$ is the stress in the SF bundle, $\sigma_{0}$ is the isometric tension, and $\overline{k_{v}}$ is the reduction in stress upon increasing the shortening strain rate, $\dot{\varepsilon}$, by $\dot{\varepsilon_{0}}$. The isometric tension $\sigma_{0}$ is specified as

$$
\sigma_{0}=\eta \sigma_{\max }
$$

where $\sigma_{\max }$ is a material property reflecting the contractility of the cell type.

The activation level, $\eta$, changes with time depending on signal intensity and fibre tension. The rate of change of the activation level of a fibre bundle is captured using a first order kinetic equation:

$$
\frac{d \eta}{d t}=(1-\eta) \frac{C \overline{k_{f}}}{\theta}-\left(1-\frac{\sigma_{f}}{\sigma_{0}}\right) \eta \frac{\overline{k_{b}}}{\theta}
$$

The first part of the equation captures SF formation in response to a signal, $C$, and the second part captures dissociation following a reduction in fibre tension below its isomteric value, as given in Equation A.2. The dimensionless rate constants $\overline{k_{f}}$ and $\overline{k_{b}}$ govern the rate of change.

Having established the behaviour of a single fibre that is oriented in an arbitrary direction (defined by the angle $\phi$ with respect to the $x_{1}$ direction; see Figure 2$)$, we can relate the fibre activation level, $\eta(\phi)$, and fibre tension, $\sigma_{f}(\phi)$, to the fibre strain rate, $\dot{\varepsilon}_{f}(\phi)$. The fibre strain rate in a given direction is obtained from the material strain rate $\dot{\varepsilon}_{\mathrm{ij}}$ by

$$
\dot{\varepsilon}_{f}=\dot{\varepsilon}_{11} \cos ^{2}(2 \phi)+\dot{\varepsilon}_{22} \sin ^{2}(\phi)+\dot{\varepsilon}_{12} \sin (2 \phi)
$$

At every point in the cell, SF formation is considered in all possible directions and the contribution of each fibre is calculated by integrating over all possible directions to give the total active stress

$$
\sigma_{i j}^{A}=\frac{1}{\pi} \int_{-\frac{\pi}{2}}^{\frac{\pi}{2}} \sigma_{f}(\phi)\left(\begin{array}{cc}
\cos ^{2} \phi & \frac{\sin 2 \phi}{2} \\
\frac{\sin 2 \phi}{2} & \sin ^{2} \phi
\end{array}\right) d \phi
$$

In parallel to the active SF behaviour described above, the passive material surrounding the SFs in the cell cytoplasm is modelled using isotropic elasticity described by a Young's modulus, $E_{c e l l}$, and Poisson's ratio, $v_{c e l l}$. The passive response includes contributions from micro-tubules etc. and provides the balancing compressive stresses as dictated by equilibrium considerations. 


\section{Adhesion model}

The cell adhesion model, which was developed by Deshpande et al. (2008), is based on the thermodynamic equilibrium of binding proteins. In the current study, this model is used to simulate integrin binding in focal adhesions and cadherin binding in cell-cell adhesions. In both cases, we can consider equilibrium between the chemical potential of bound and unbound adhesion proteins with concentrations $\xi_{B}$ and $\xi_{U}$, respectively such that

$$
\mu_{B}+k \operatorname{Tln}\left(\frac{\xi_{B}}{\xi_{0}}\right)+\Phi(\Delta)-F_{i}^{b} \Delta_{i}=\mu_{U}+k T \ln \left(\frac{\xi_{U}}{\xi_{0}}\right)
$$

where $\xi_{0}$ is the total concentration of binders, $\mu_{B}$ and $\mu_{U}$ are the reference chemical potentials of the bound and unbound adhesion proteins. Here, $k$ and $T$ are the Boltzmann constant and the absolute temperature, respectively. The stretch energy of the adhesion bond is denoted by $\Phi$ and $F_{i}^{b} \Delta_{i}$ is the mechanical work due to the stretch $\Delta$ of the bond by the force $F_{i}^{b} \equiv \partial \Phi / \partial \Delta_{i}$. The concentrations of bound and unbound proteins is then given by

$$
\begin{aligned}
\xi_{B} & =\frac{\xi_{0}}{\exp \left[\frac{\mu_{B}-\mu_{U}+\Phi-F_{b_{i}} \Delta_{i}}{k T}\right]+1} \\
\xi_{U} & =\frac{\xi_{0}}{\exp \left[-\frac{\mu_{B}-\mu_{U}+\Phi-F_{b_{i}} \Delta_{i}}{k T}\right]+1}
\end{aligned}
$$

respectively. The stretch energy $\Phi$ is expressed as a piecewise quadratic potential:

$$
\Phi=\left\{\begin{array}{cc}
\kappa_{s} \Delta_{e}^{2} & \Delta_{e} \leq \Delta_{n} \\
-\kappa_{s} \Delta_{n}^{2}+2 \kappa_{s} \Delta_{n} \Delta_{e}-\left(\kappa_{s} \Delta_{e}^{2}\right. & \Delta_{n}<\Delta_{e} \leq 2 \Delta_{n} \\
\kappa_{s} \Delta_{n}^{2} & \Delta_{e}>2 \Delta_{n}
\end{array}\right.
$$

where $\kappa_{s}$ is the stiffness of the bond; $\Delta_{e} \equiv \sqrt{\Delta_{1}^{2}+\Delta_{2}^{2}}$ is the stretch magnitude and $\Delta_{n}$ is the peak bond length.

In the case of FAs, binding takes place between integrins on the cell surface and ligands on the posts while in the case of cell-cell adhesion, cadherin from each cell binds to cadherin in the neighbouring cell. The above adhesion model is used in both circumstances.

\section{Micro-post model and equilibrium}

The tractions $T_{i}$ on the cell surface depend on the force $F_{i}^{b}$ on each bond and the concentration of bound proteins. These tractions are balanced by stresses in the cell resulting from cellular contractility such that 


$$
T_{i}=\sigma_{i j} n_{j}=-\xi_{B} F_{i}^{b}
$$

where $\sigma_{i j}$ is the Cauchy stress in the cell, and $n_{j}$ is the surface normal. In the case of FAs, the cell exerts a traction on the post causing the post to deflect. The micro-posts behave as vertical cantilevers which are completely fixed at the base and subjected to loading only at the post tip. Thus, we can relate the force $\left(F_{p}\right)$ applied to the micro-post surface to the tip deflection $\left(\delta_{p}\right)$ as:

$$
\boldsymbol{F}_{p}=\left(\frac{3 E_{p} I_{p}}{L_{p}^{3}}\right) \boldsymbol{\delta}_{p} \equiv k_{p} \boldsymbol{\delta}_{p}
$$

where $E_{p}$ is the Young's modulus of the post material (PDMS), $I_{p}$ is the second moment of area of the post cross-section about its diametrical axis, and $L_{p}$ is the length (or height) of the post. The rotation of the micro-post surface with respect to long axis of the post is proportional to the ratio $\left|\boldsymbol{\delta}_{p}\right| / L_{p}$, thus where $\left|\boldsymbol{\delta}_{p}\right|$ is small compared to the length of the micro-post, we can neglect the rotation of this surface. Therefore, it is sufficient to consider the posts as rigid circular surfaces attached to a spring which represents the bending stiffness of the beam, $k_{p}$, i.e. term in parentheses in Equation A.12. In Liu et al. (2010) the PDMS micro-posts of length of $11 \mu \mathrm{m}$ and radius of $1.5 \mu \mathrm{m}$ resulted in $k_{p}=32$ $\mathrm{nN} / \mu \mathrm{m}$. 
Tables

Table 1: Cell contractility parameters for each cell phenotype.

\begin{tabular}{cllll}
\hline & SMC & MSC & FB & ETC \\
\hline $\boldsymbol{\sigma}_{\text {max }}$ & $25 \mathrm{kPa}$ & $8 \mathrm{kPa}$ & $3.5 \mathrm{kPa}$ & $2.0 \mathrm{kPa}$ \\
$\overline{\boldsymbol{k}_{\boldsymbol{v}}}$ & 7 & 12 & 7 & 20 \\
& & & & \\
\hline
\end{tabular}




\section{Figure captions}
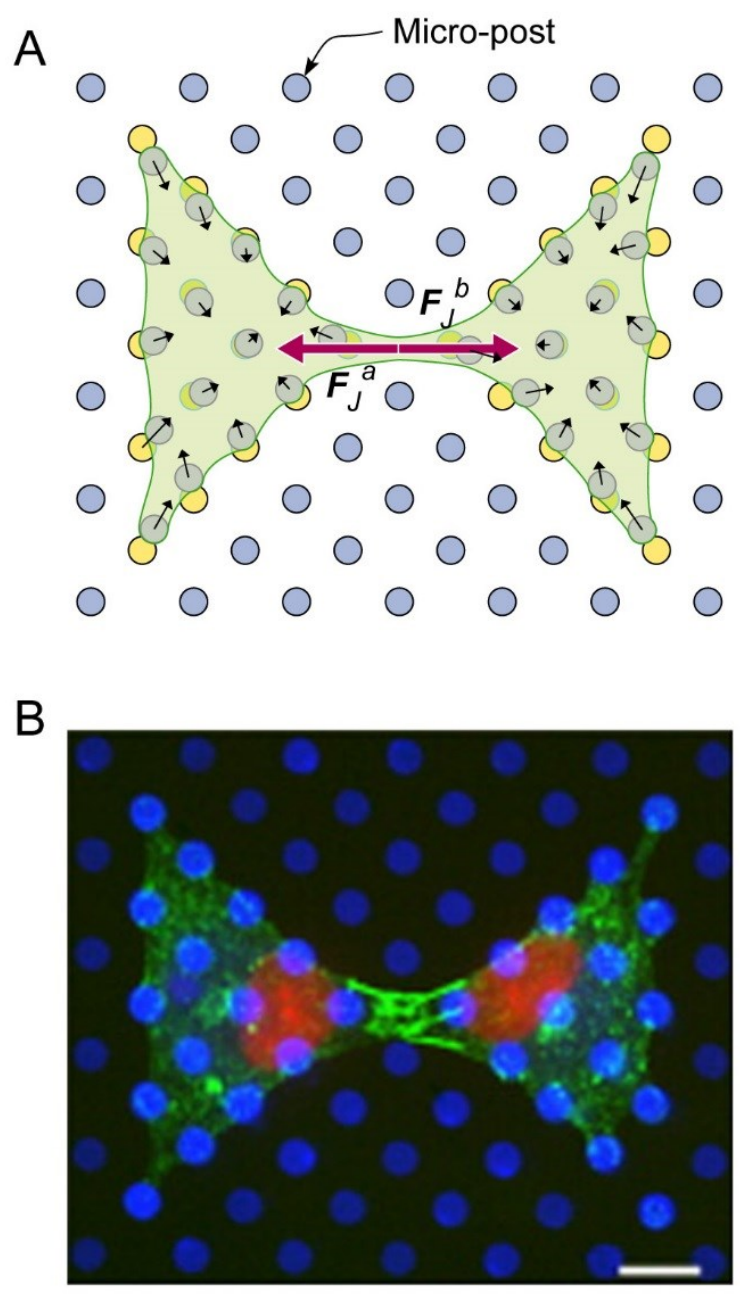

Figure 1: Experimental setup used by Liu et al. (2010) to measure cell-cell tugging forces across cell-cell junctions (A) Fluorescence microscopy of cell-cell junctions (green), reproduced from Liu et al. (2010) (B). Scale bar $10 \mu \mathrm{m}$. 
A

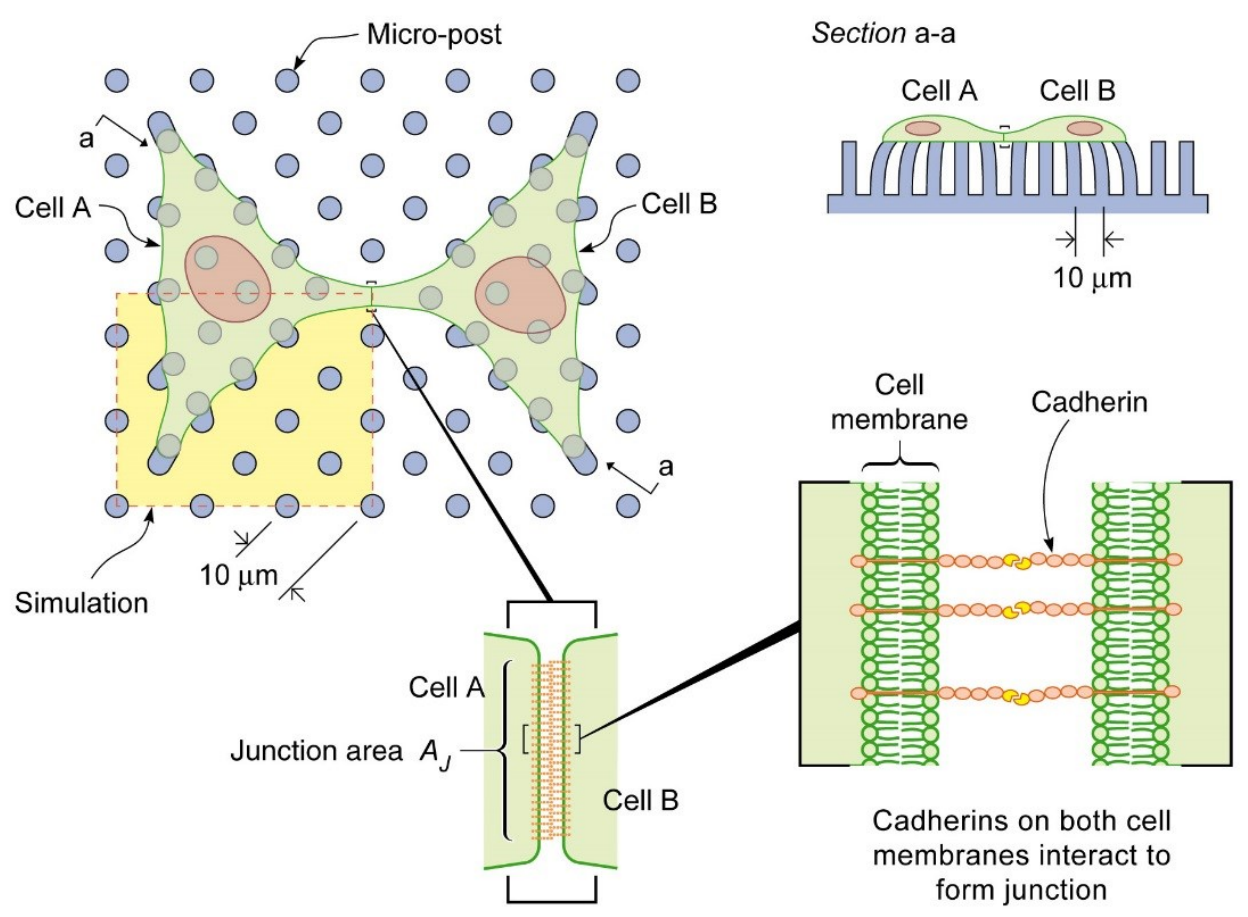

B

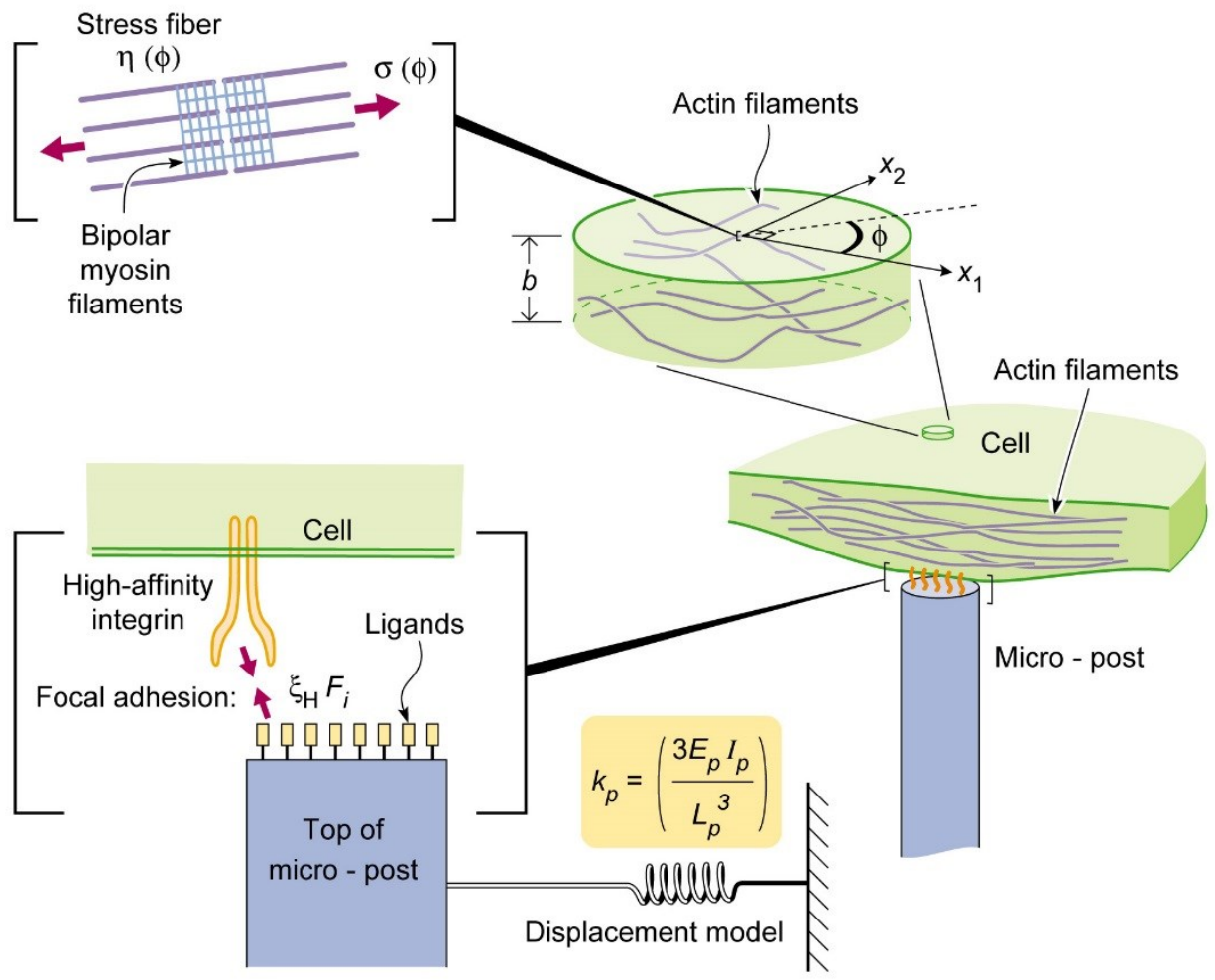

Figure 2: Sketch of the finite element model with two cells adhered to an array of micro-posts (A). Using symmetry it is sufficient to model the area marked in yellow by the dashed line. The micro-posts are modelled as linear springs and the spring constants represent the stiffness of the micro-posts (B). Focal adhesions attach the cell to the micro-posts. 
A
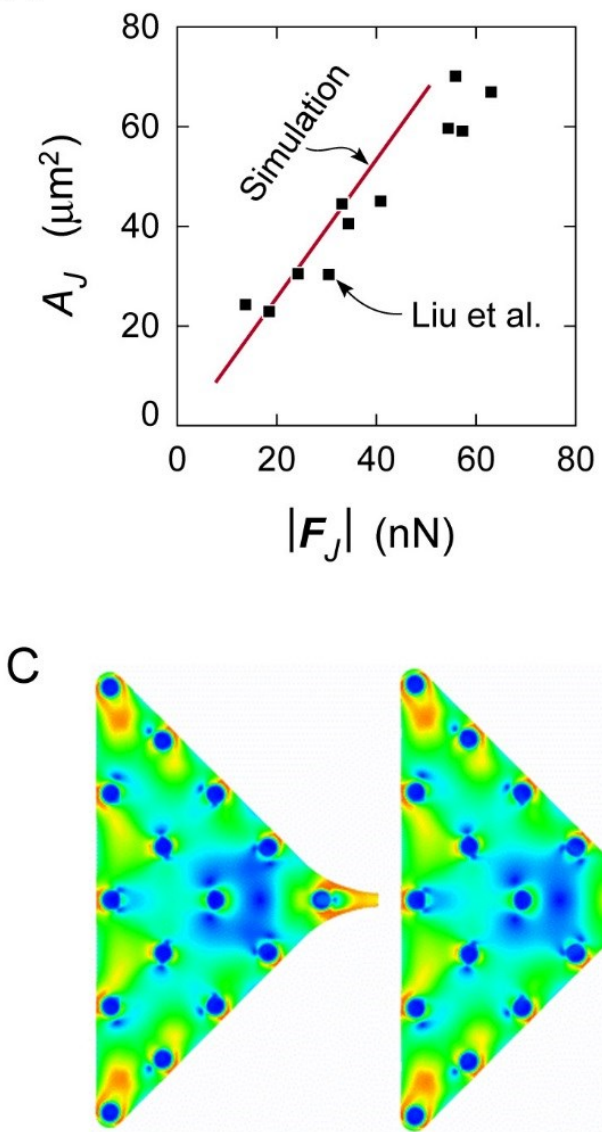

$A_{J}=12.5$

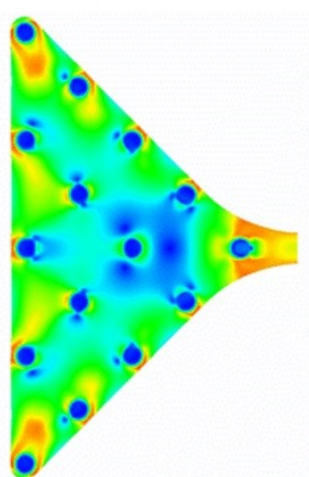

25
B

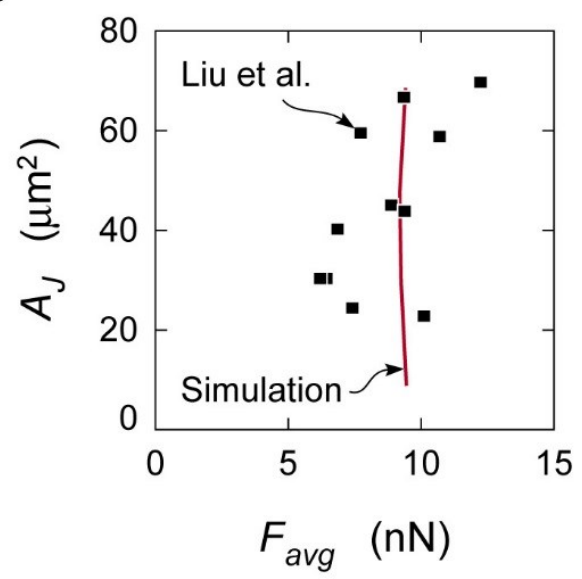

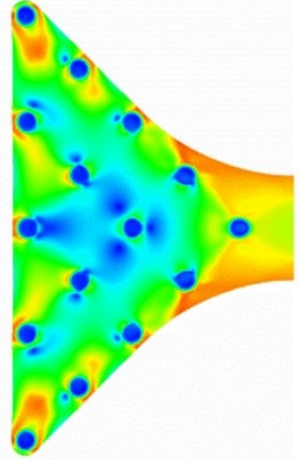

$75 \mu \mathrm{m}^{2}$

$\Pi$\begin{tabular}{llll|l|l|l}
0.0 & 0.2 & 0.4 & 0.6 & 0.8
\end{tabular}

Figure 3: The simulations of cell pairs on micro-post arrays capture two phenomena, as observed experimentally by Liu et al. (2010): adhesion junction size $A_{J}$ is linearly correlated with junction force $\left|F_{J}\right|(\mathrm{A})$; adhesion junction size is not correlated with average traction force $F_{\text {avg }}(\mathrm{B})$. The experimental results are plotted here (black squares) and superimposed over simulated results (solid red lines). Predicted distributions of stress fibres (SFs) are shown for cell pairs forming junctions with different cross sectional areas $A_{J}(\mathrm{C})$. Note: only one cell of the symmetric pair is shown, with the junction on the right of each cell. 
A

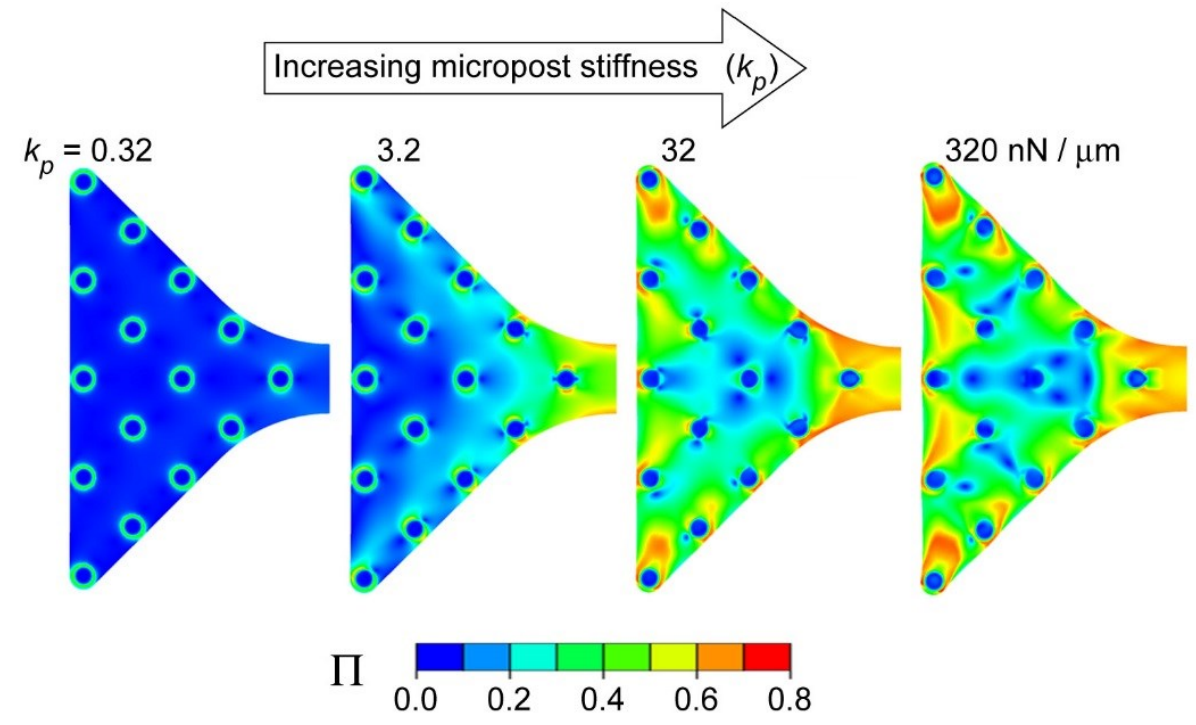

B

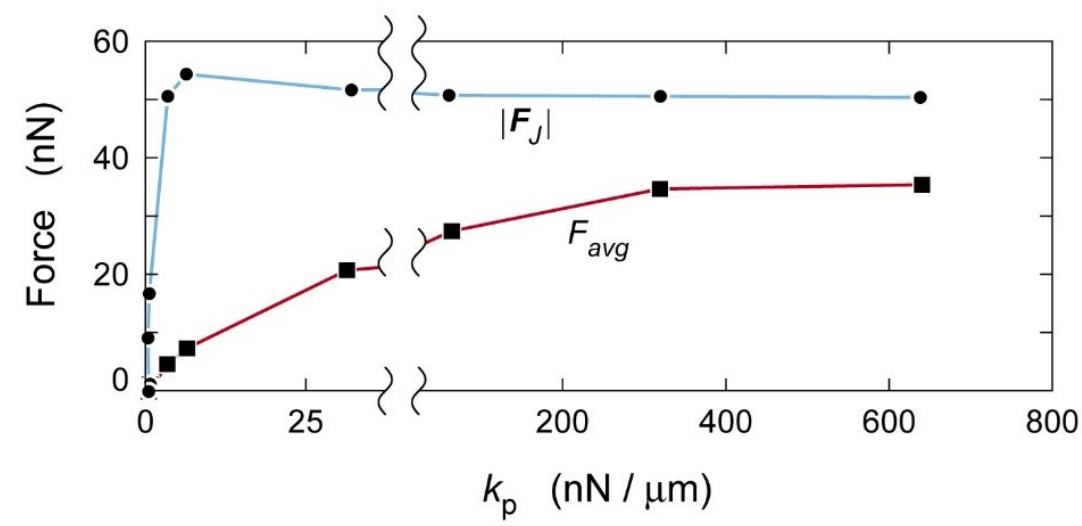

C

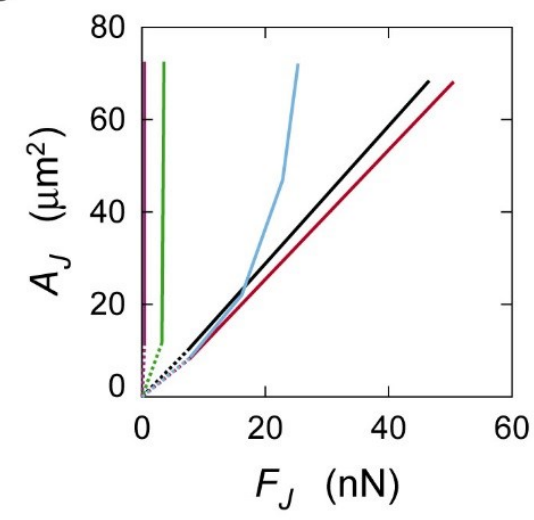

D

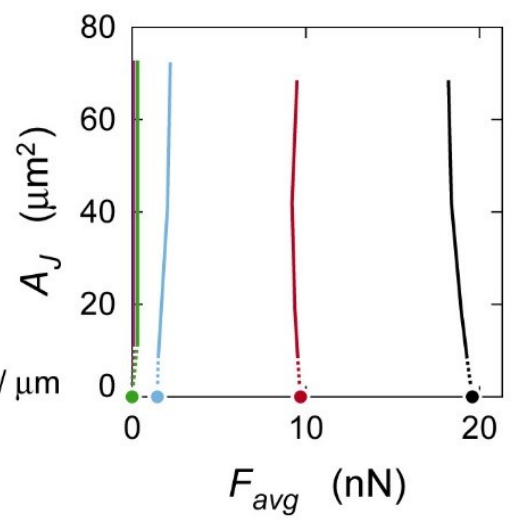

Figure 4: Stress fibre (SF) formation, as parameterised by $\Pi$, for bowtie endothelial cell pairs adhered to micro-post array with different micro-post stiffness $k_{p}(\mathrm{~A})$. Note that only one cell from the symmetric pair is shown. Increasing micro-post stiffness causes a monotonic increase in average traction force, $F_{\text {avg }}$ (red), and also causes a sharp increase in junction tugging force, $\left|F_{J}\right|$ (blue), up to $3.2 \mathrm{nN} / \mu \mathrm{m}$ followed by a plateau in tugging force for stiffer micro-posts (B). The linear dependence of junction tugging force $\left|F_{J}\right|$ on junction size $A_{J}$ disappears for very compliant micro-posts $(<1 \mathrm{nN} / \mu \mathrm{m})$ but is preserved for very stiff micro-posts $(\mathrm{C})$. Average traction $F_{\text {avg }}$ is found to be independent of junction size for all micro-posts stiffness (D). In (D) predictions for a single triangular cell that forms one-half of the bowtie are included for a single cell with no junction, corresponding to $A_{J}=0$. 

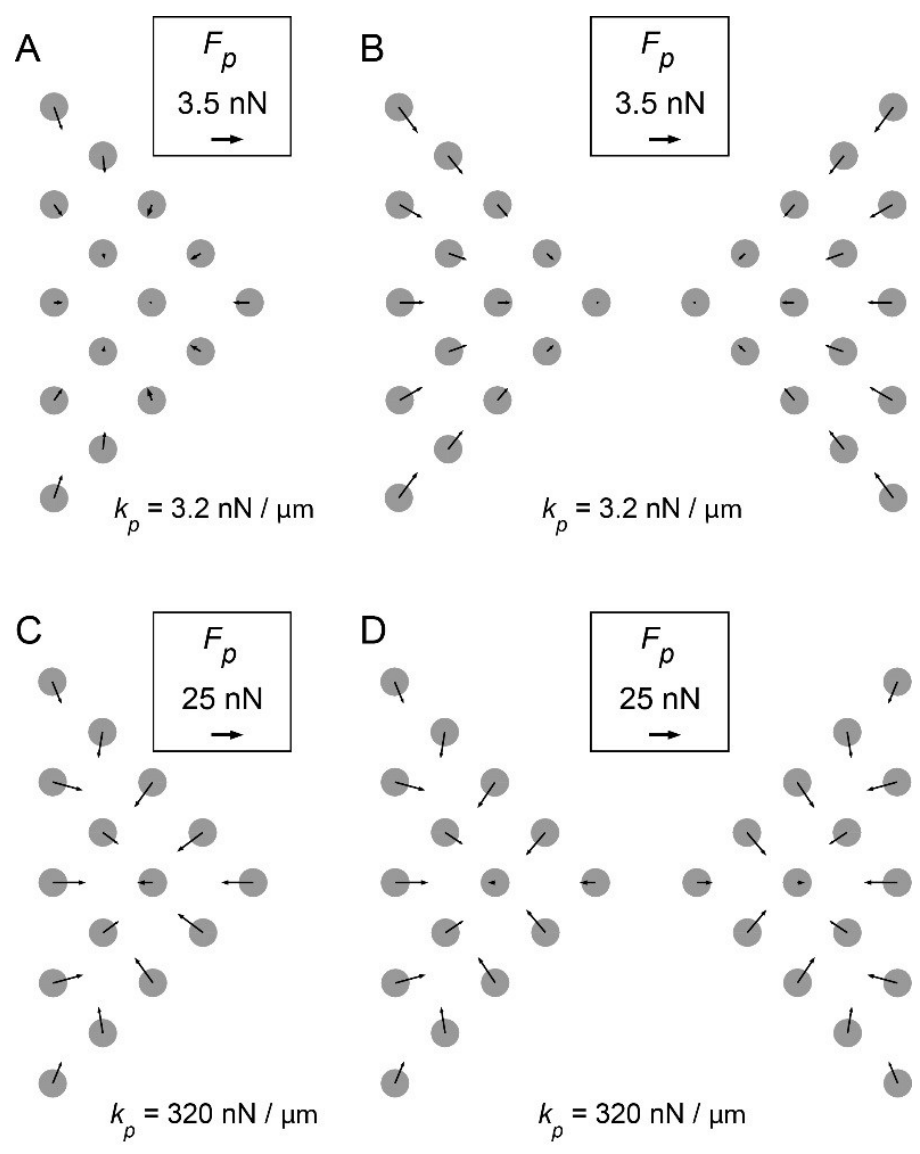

Figure 5: Predicted post forces $\left(F_{p}\right)$ for single cells $(A, C)$ and cell pairs $(B, D)$ for $3.2 \mathrm{nN} / \mu \mathrm{m}(A, B)$ and $320 \mathrm{nN} / \mu \mathrm{m}(C, D)$ micro-posts. The cell pairs (B,D) have an junction area $A_{J}$ of $50 \mu \mathrm{m}^{2}$. Scale bar for post forces $\left(F_{p}\right)$ are shown in each case. 
A

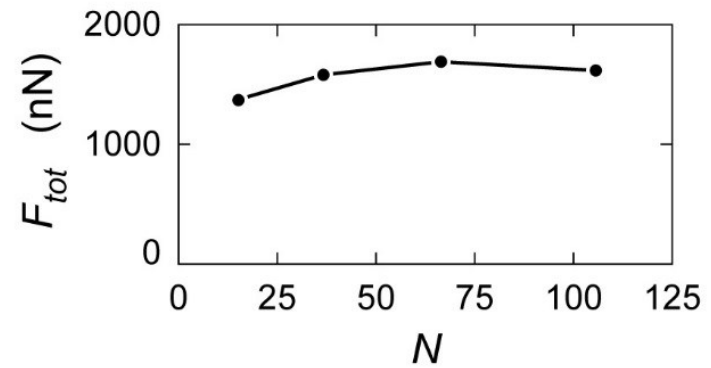

D

$\mathrm{B}$
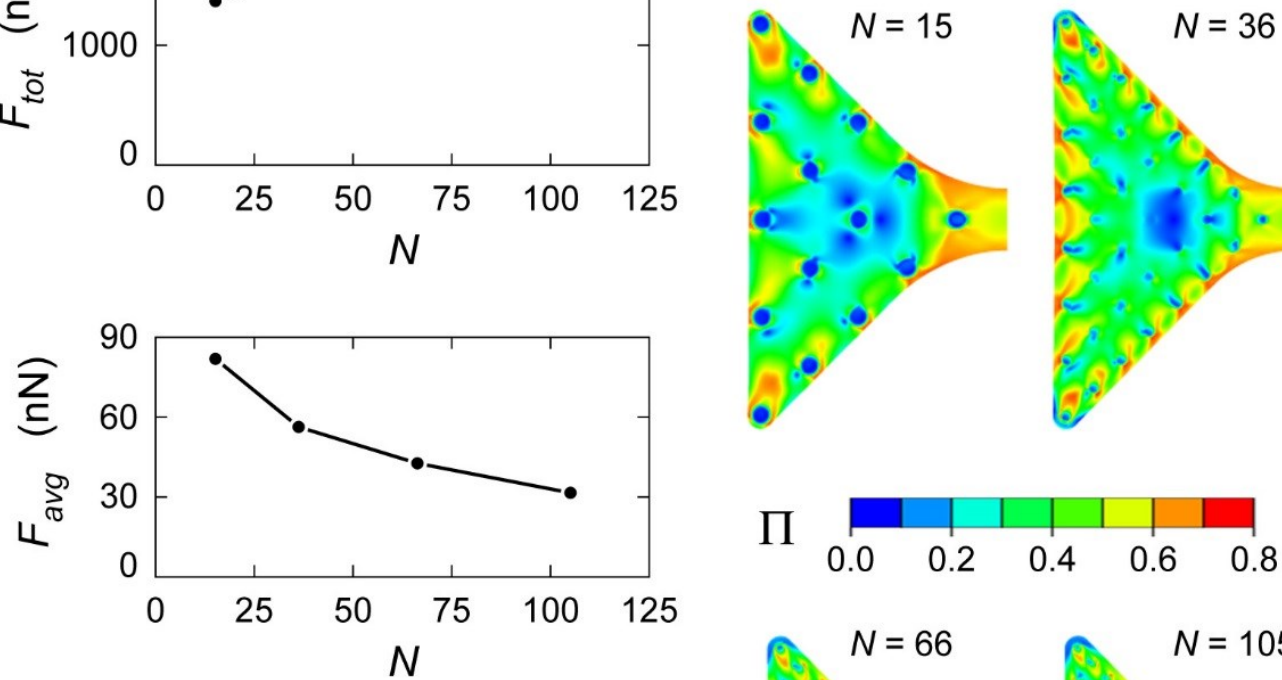

C
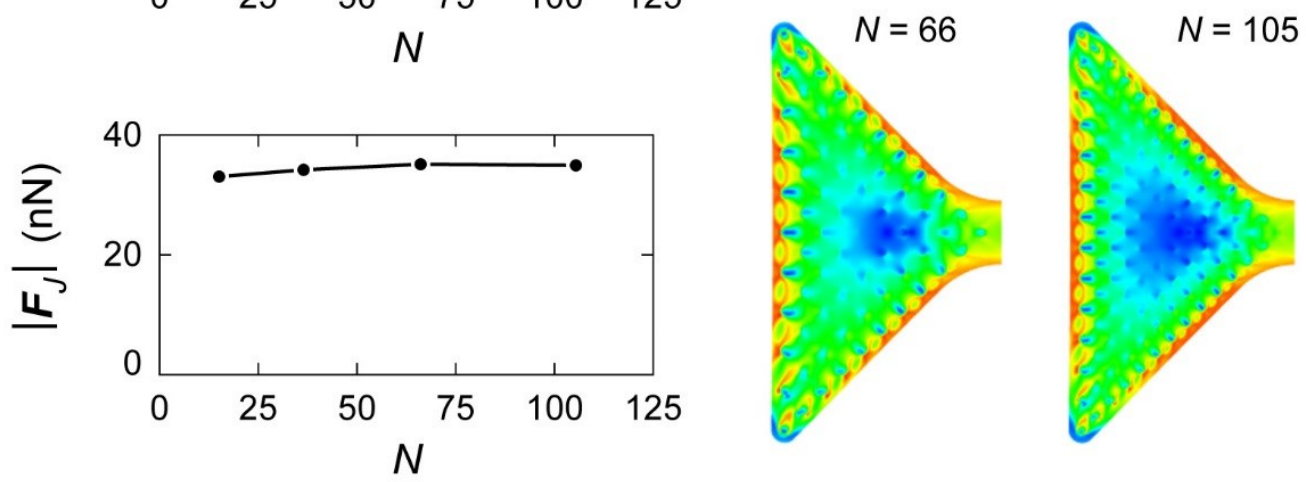

Figure 6: Total micro-post tractions $F_{\text {tot }}$ and junction tugging force $\left|F_{J}\right|$ are unchanged by number of adhered micro-posts $(A, C)$. Average traction $F_{a v g}$ decreases significantly with increasing number of micro-posts $(B)$. Stress fibre distributions are largely unaffected by number of adhered micro-posts $(D)$. The centre to centre spacing of the posts and post diameter are adjusted such that the projected cell area and total micro-post area are kept constant. 
A
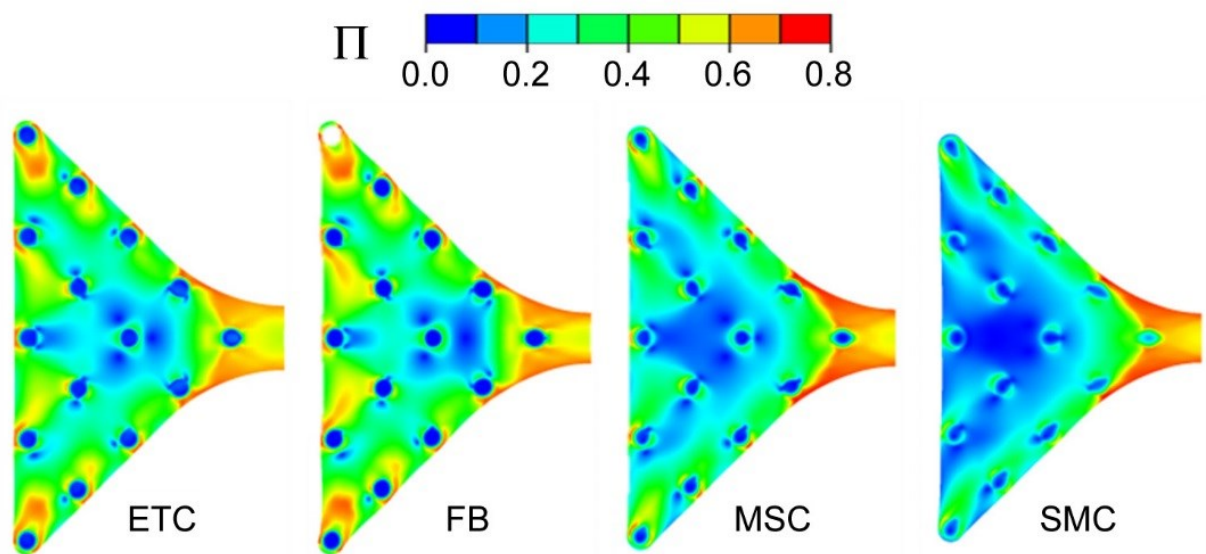

B

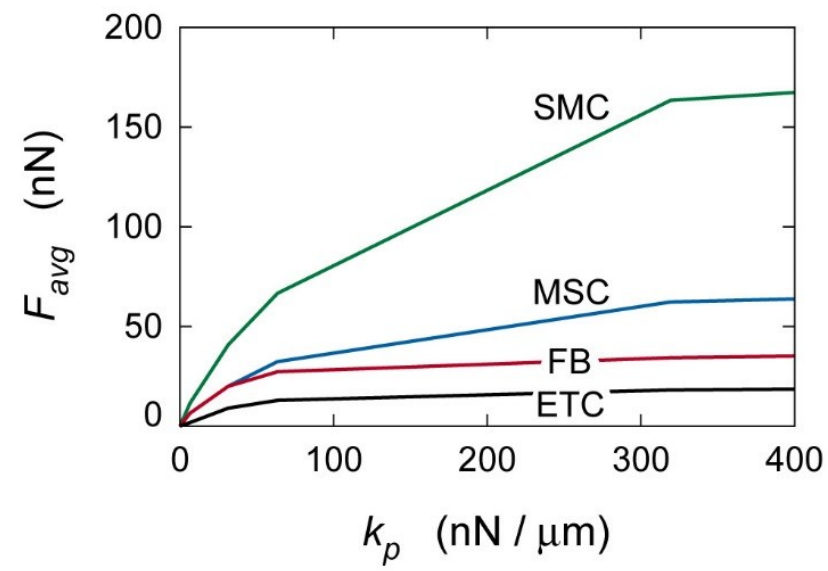

C

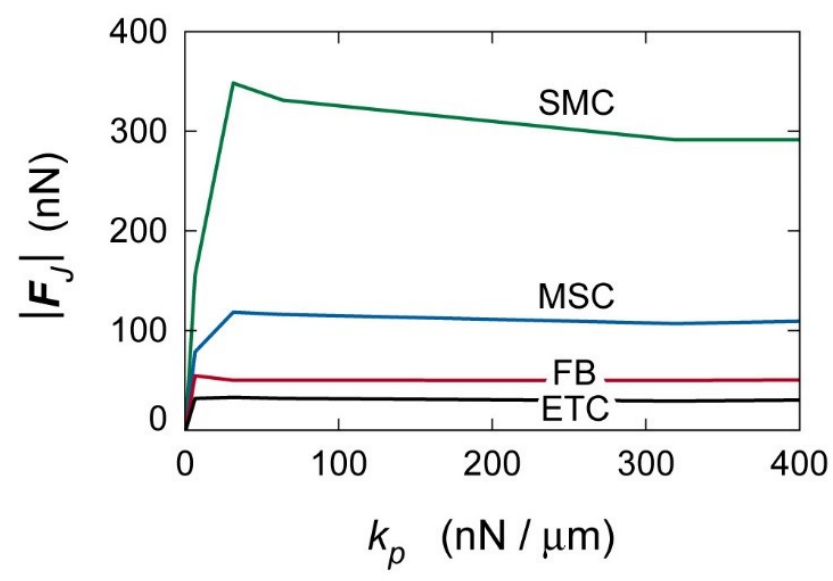

Figure 7: Predicted stress fibre (SF) formation in bowtie cell pairs for endothelial cells (ETC), fibroblasts (FB), mesenchymal stem cells (MSC), and smooth muscle cells (SMC) (A). SFs are shown for one cell of a symmetric pair seeded on $32 \mathrm{nN} / \mu \mathrm{m}$ micro-posts with a $50 \mu \mathrm{m}^{2}$ junction area. Average traction $F_{\text {avg }}$ increases with increasing micro-post stiffness for all cell types (B). Junction tugging force $\left|F_{J}\right|$ shows no change with micro-post stiffness for a wide range of stiffness for all cell types (C). 